\title{
Constraining Dark Matter-Dark Energy Interaction with Gas Mass Fraction in Galaxy Clusters
}

\author{
R. S. Gonçalves ${ }^{1}$, J. S. Alcaniz ${ }^{1}$ A. Dev ${ }^{2}$ and D. Jain ${ }^{3}$ \\ ${ }^{1}$ Observatório Nacional, \\ Rio de Janeiro - RJ, 20921-400, Brasil \\ ${ }^{2}$ Deen Dayal Upadhyaya College, \\ University of Delhi, Delhi - 110015, India \\ ${ }^{3}$ Miranda House, \\ University of Delhi, Delhi - 110007, India
}

\begin{abstract}
The recent observational evidence for the current cosmic acceleration have stimulated renewed interest in alternative cosmologies, such as scenarios with interaction in the dark sector (dark matter and dark energy). In general, such models contain an unknown negative-pressure dark component coupled with the pressureless dark matter and/or with the baryons that results in an evolution for the Universe rather different from the one predicted by the standard $\Lambda$ CDM model. In this work we test the observational viability of such scenarios by using the most recent galaxy cluster gas mass fraction versus redshift data (42 X-ray luminous, dynamically relaxed galaxy clusters spanning the redshift range $0.063<z<1.063$ ), Allen et al. (2008), to place bounds on the parameter $\epsilon$ that characterizes the dark matter/dark energy coupling. The resulting are consistent with, and typically as constraining as, those derived from other cosmological data. Although a time-independent cosmological constant $(\Lambda \mathrm{CDM}$ model) is a good fit to these galaxy cluster data, an interacting energy component cannot yet be ruled out.
\end{abstract}

Keywords. Cosmology - Cosmological Parameters - Coupled Quintessence - Distance Scale Galaxy Clusters.

\section{References}

Allen, S. W. et al., MNRAS, 383 (2008) 